\title{
EULER'S FACTORIAL SERIES AT ALGEBRAIC INTEGER POINTS
}

\author{
LOUNA SEPPÄLÄ
}

\begin{abstract}
We study a linear form in the values of Euler's series $F(t)=\sum_{n=0}^{\infty} n ! t^{n}$ at algebraic integer points $\alpha_{1}, \ldots, \alpha_{m} \in \mathbb{Z}_{\mathbb{K}}$ belonging to a number field $\mathbb{K}$. Let $v \mid p$ be a non-Archimedean valuation of $\mathbb{K}$. Two types of non-vanishing results for the linear form $\Lambda_{v}=\lambda_{0}+\lambda_{1} F_{v}\left(\alpha_{1}\right)+\ldots+\lambda_{m} F_{v}\left(\alpha_{m}\right), \lambda_{i} \in \mathbb{Z}_{\mathbb{K}}$, are derived, the second of them containing a lower bound for the $v$-adic absolute value of $\Lambda_{v}$. The first non-vanishing result is also extended to the case of primes in residue classes. On the way to the main results, we present explicit Padé approximations to the generalised factorial series $\sum_{n=0}^{\infty}\left(\prod_{k=0}^{n-1} P(k)\right) t^{n}$, where $P(x)$ is a polynomial of degree one.
\end{abstract}

\section{IntRoduction}

Euler's factorial series

$$
F(t):={ }_{2} F_{0}(1,1 \mid t)=\sum_{n=0}^{\infty} n ! t^{n}
$$

converges $p$-adically for all primes $p$ when $|t|_{p} \leq 1$. In the $v$-adic metric (where $v$ extends $p$ for some prime $p$ ) of a number field $\mathbb{K}$, the series $F(t)$ converges to a point in the $v$-adic closure of $\mathbb{K}$ (denoted by $\mathbb{K}_{v}$ ) when $t \in \mathbb{K}$ is such that $|t|_{v}<p^{\frac{1}{p-1}}$. Thus we write $\sum_{n=0}^{\infty} n ! t^{n}=: F_{v}(t)$ when treating the series as a function in the $v$-adic domain $\mathbb{K}$.

Euler's series (11) is a member of the class of $F$-series (series of the form $\sum_{n=0}^{\infty} a_{n} n ! z^{n}$, with certain conditions on the coefficients $a_{n}$ ) introduced by V. G. Chirskil in [3, 4]. In those papers he answered the problem of the existence of global relations 11 between members of the class of $F$-functions. As he points out in [5], the results can be refined in terms of estimating the prime $p$ for which there exists a valuation $v \mid p$ breaking the global relation. These estimates were made entirely effective by Bertrand, Chirskiı, and Yebbou in [2]. In [2, Theorem 1.1] they describe an infinite collection of intervals each containing a prime number $p$ such that for some valuation $v \mid p$ it holds

$$
h_{1} f_{1}(\xi)+\ldots+h_{m} f_{m}(\xi) \neq 0,
$$

where $h_{i} \in \mathbb{Z}_{\mathbb{K}}$ and $f_{1}(t) \equiv 1, f_{2}(t), \ldots, f_{m}(t)$ are $F$-series that are linearly independent over $\mathbb{K}(z)$ and constitute a solution to a differential system $D$, and $\xi \in \mathbb{K} \backslash\{0\}$ is an ordinary point of the system $D$. What is more, the non-vanishing in (2) is replaced by a lower bound for $\left|h_{1} f_{1}(\xi)+\ldots+h_{m} f_{m}(\xi)\right|_{v}$.

Date: October 1, 2018.

2010 Mathematics Subject Classification. 11J61, 41A21.

Key words and phrases. Diophantine approximation, divergent series, number fields, Padé approximation, $p$-adic, valuations.

${ }^{1}$ Let $P \in \mathbb{K}\left[x_{1}, \ldots, x_{m}\right]$ be a polynomial of $m$ variables and suppose $F_{1}(t), \ldots, F_{m}(t) \in \mathbb{K}[[t]]$ are power series. Take a $\xi \in \mathbb{K}$. A relation

$$
P\left(F_{1}(\xi), \ldots, F_{m}(\xi)\right)=0
$$

is called global if it holds in all the fields $\mathbb{K}_{v}$ where all the series $F_{1}(\xi), \ldots, F_{m}(\xi)$ converge. 
In their recent paper [11, T. Matala-aho and W. Zudilin studied the irrationality of $F_{p}(\xi)$ at a point $\xi \in \mathbb{Z} \backslash\{0\}$ (i.e. global relations of the numbers 1 and $F_{p}(\xi)$ ). In Theorem 3.1 of this paper we generalise their idea to a linear form

$$
\Lambda_{v}:=\lambda_{0}+\lambda_{1} F_{v}\left(\alpha_{1}\right)+\ldots+\lambda_{m} F_{v}\left(\alpha_{m}\right), \quad \lambda_{i} \in \mathbb{Z}_{\mathbb{K}},
$$

in values of Euler's series at $m$ given pairwise distinct algebraic integer points $\alpha_{1}, \ldots, \alpha_{m} \in$ $\mathbb{Z}_{\mathbb{K}} \backslash\{0\}$. Theorem 3.1 states that in any collection $V$ of non-Archimedean valuations of $\mathbb{K}$ satisfying a certain condition, there exists a valuation $v^{\prime} \in V$ such that $\Lambda_{v^{\prime}} \neq 0$. The result can also be extended to the case of primes in arithmetic progressions, generalising the recent result of [8]. This is done in Theorem 9.6 of Section 9 ,

In the second main result, Theorem 3.4, we characterise an interval $I(m, H)$ (where $H$ is an upper bound for the height of the coefficients $\lambda_{i}$ ) from which one can find a prime $p$ such that there exists a valuation $v^{\prime} \mid p$ for which

$$
\left\|\Lambda_{v^{\prime}}\right\|_{v^{\prime}}>H^{-(m+1)-114 m^{2} \cdot \frac{\log \log \log H}{\log \log H}} .
$$

Our method is based on explicit Padé approximations, whereas Bertrand, Chirskiŭ, and Yebbou [2] use Siegel's lemma. In addition, the functional dependence on $H$ in our lower bound is improved compared to [2].

The proofs of both our main results rely on Padé approximations which are used to construct small approximation forms for the values $F_{v}\left(\alpha_{j}\right), j=1, \ldots, m$. Therefore, before moving to the proofs of the theorems, we shall present explicit Padé approximations (with the orders of the remainders as free parameters) to the generalised factorial series

$$
G(t)=\sum_{n=0}^{\infty}[P]_{n} t^{n},
$$

where $P(x)$ is a polynomial of degree one and $[P]_{n}:=\prod_{k=0}^{n-1} P(k)$ (see Theorem 4.2).

A brief outline of the proofs is presented right after the formulation of the main results in Section 3. In addition to Theorem 9.6, the last section contains some examples of the use of the main results. We shall study the sum

$$
\sum_{n=0}^{\infty} n ! f_{n},
$$

where $\left(f_{n}\right)_{n=0}^{\infty}$ is the sequence of Fibonacci numbers, and show that for any rational number $\frac{a}{b} \in \mathbb{Q}$ there exists a valuation of the field $\mathbb{Q}(\sqrt{5})$ such that

$$
\sum_{n=0}^{\infty} n ! f_{n} \neq \frac{a}{b} \text {. }
$$

\section{Preliminaries: Number fields and Valuations}

Let $\mathbb{K}=\mathbb{Q}(\gamma)$ be an algebraic number field of degree $\kappa$, and let $\mathbb{Z}_{\mathbb{K}}$ be its ring of integers (the algebraic integers contained in $\mathbb{K}$ ). All the absolute values of $\mathbb{K}$ are extensions of the absolute values of $\mathbb{Q}$. When $p \in \mathbb{P} \cup\{\infty\}$, where $\mathbb{P}$ is the set of prime numbers, there are as many distinct extensions of $|\cdot|_{p}$ to $\mathbb{K}$ as there are irreducible factors of the minimal polynomial of $\gamma$ in $\mathbb{Q}_{p}[x]$ (see [1, Chapter V]). Here $\mathbb{Q}_{p}$ denotes the topological closure of $\mathbb{Q}$ with respect to the metric $|\cdot|_{p}$, so that $\mathbb{Q}_{\infty}=\mathbb{R}$.

If $|\cdot|_{v}$ extends the standard $p$-adic metric $|\cdot|_{p}$ to $\mathbb{K}$, it is customary to write $v \mid p$, and similarly, when extending the Archimedean absolute value $|\cdot|=|\cdot|_{\infty}$, we write $v \mid \infty$. The collection of non-Archimedean valuations of $\mathbb{K}$ is denoted by $V_{0}$, and the collection of Archimedean valuations of $\mathbb{K}$ by $V_{\infty}$. 
The topological closure of $\mathbb{K}$ with respect to the metric $|\cdot|_{v}$ is denoted by $\mathbb{K}_{v}$. We also denote $\kappa_{v}=\left[\mathbb{K}_{v}: \mathbb{Q}_{p}\right]$ (local degree), so that $\sum_{v} \kappa_{v}=\kappa=[\mathbb{K}: \mathbb{Q}]$.

2.1. Normalisation. If $v \mid p$ for a prime $p$, there is an element $\pi \in \mathbb{K}$ such that $|\pi|_{v}<1$ and $\left\langle|\pi|_{v}\right\rangle=|\mathbb{K} \backslash\{0\}|_{v}$. Then $p=u \pi^{e}$, where $u$ is a unit of $\mathbb{K}$ and $e=e_{v}(\mathbb{K}, \mathbb{Q})=\left[|\mathbb{K}|_{v}\right.$ : $\left.|\mathbb{Q}|_{v}\right]$ is the ramification index of the extension. It follows naturally from $|p|_{p}=\frac{1}{p}$ that

$$
|p|_{v}=\frac{1}{p}, \quad|\pi|_{v}=\frac{1}{p^{\frac{1}{e}}}
$$

However, it is convenient to use the normalisation

$$
\|p\|_{v}=|p|_{v}^{\frac{\kappa_{v}}{k}}, \quad\|\pi\|_{v}=|\pi|_{v}^{\frac{\kappa_{v}}{k}}
$$

Similarly, if $v \mid \infty$ and corresponds to the $i$ th conjugate field $\mathbb{K}^{(i)}$, then we set

$$
\|x\|_{v}=\left|x^{(i)}\right|^{\frac{\kappa_{v}}{\kappa}},
$$

where $x^{(i)}$ is the $i$ th conjugate of $x \in \mathbb{K}$. Since $\frac{\kappa_{v}}{\kappa} \leq 1$, the triangle inequality is valid also for the normalised Archimedean absolute value.

2.2. Product formula. The following product formula holds for any $x \in \mathbb{K} \backslash\{0\}$ :

$$
\prod_{v}\|x\|_{v}=1
$$

where the product is taken over all normalised, pairwise non-equivalent valuations of $\mathbb{K}$. Note that if $x \in \mathbb{Q}$, then

$$
\prod_{v \mid p}\|x\|_{v}=|x|_{p}
$$

for any $p \in \mathbb{P} \cup\{\infty\}$.

For more details on valuations, the reader is advised to consult [1] and [10].

\section{Results}

Let $m \in \mathbb{Z}_{\geq 1}$ and choose $m$ pairwise distinct, non-zero algebraic integers $\alpha_{1}, \ldots, \alpha_{m} \in$ $\mathbb{Z}_{\mathbb{K}} \backslash\{0\}$. Denote $\bar{\alpha}=\left(\alpha_{1}, \ldots, \alpha_{m}\right)^{T}$. We define

$$
c_{1}=c_{1}(\bar{\alpha})=\prod_{v \in V_{\infty}}\left(\left(\max _{1 \leq j \leq m}\left\{1,\left\|\alpha_{j}\right\|_{v}\right\}\right)^{m} \prod_{i=1}^{m}\left(\left\|\alpha_{i}\right\|_{v}+\max _{1 \leq j \leq m}\left\{1,\left\|\alpha_{j}\right\|_{v}\right\}\right)\right)
$$

and

$$
c_{2}=c_{2}(\bar{\alpha}, V)=c_{1} \prod_{v \in V} \max _{1 \leq j \leq m}\left\{\left\|\alpha_{j}\right\|_{v}\right\}
$$

for any $V \subseteq V_{0}$

Theorem 3.1. Let $\lambda_{0}, \lambda_{1}, \ldots, \lambda_{m} \in \mathbb{Z}_{\mathbb{K}}$ be such that $\lambda_{j} \neq 0$ for at least one $j$. Suppose $V \subseteq V_{0}$ is a collection of non-Archimedean valuations of $\mathbb{K}$ such that

$$
\limsup _{l \rightarrow \infty} c_{2}^{l}(m l+m)^{\kappa}(m l+m) ! \prod_{v \in V}\|(m l) ! l !\|_{v}=0 .
$$

Then there exists a valuation $v^{\prime} \in V$ for which

$$
\lambda_{0}+\lambda_{1} F_{v^{\prime}}\left(\alpha_{1}\right)+\ldots+\lambda_{m} F_{v^{\prime}}\left(\alpha_{m}\right) \neq 0 .
$$


Remark 3.2. Let us show that any collection $V \subseteq V_{0}$ whose complement in $V_{0}$ is finite satisfies condition (6). Choose $v_{1}, \ldots, v_{k} \in V_{0}$ and let $V=V_{0} \backslash\left\{v_{1}, \ldots, v_{k}\right\}$. Suppose in addition that $v_{i} \mid p_{i}$ for some $p_{i} \in \mathbb{P}, i=1, \ldots, k$. Then, by recalling that

$$
|n !|_{p} \geq p^{-\frac{n}{p-1}}
$$

and using the product formula (44), we get

$$
\begin{aligned}
& c_{2}^{l}(m l+m)^{\kappa}(m l+m) ! \prod_{v \in V}\|(m l) ! l !\|_{v} \\
& =\frac{c_{2}^{l}(m l+m)^{\kappa}(m l+m) !}{\left(\prod_{i=1}^{k}\|(m l) ! l !\|_{v_{i}}\right) \prod_{v \in V_{\infty}}\|(m l) ! l !\|_{v}} \\
& =\frac{c_{2}^{l}(m l+m)^{\kappa}(m l+m) !}{\left(\left.\prod_{i=1}^{k}|(m l) ! l !|\right|_{p_{i}} ^{\frac{\kappa v_{i}}{\kappa}}\right)(m l) ! l !} \\
& \leq \frac{c_{2}^{l}\left(\prod_{i=1}^{k} p_{i}^{\frac{\kappa v_{i}}{\kappa} \cdot \frac{m l+l}{p_{i}-1}}\right)(m l+m)^{\kappa}(m l+m) !}{(m l) ! l !} \\
& =\frac{\left(c_{2} \prod_{i=1}^{k} p_{i}^{\frac{\kappa v_{i}}{\kappa} \cdot \frac{m+1}{p_{i}-1}}\right)^{l}(m l+m)^{\kappa}(m l+1) \cdots(m l+m)}{l !} \stackrel{\rightarrow \rightarrow \infty}{\rightarrow} 0 .
\end{aligned}
$$

Remark 3.3. From the previous remark it follows that there are infinitely many valuations $v \in V_{0}$ such that $\Lambda_{v} \neq 0$.

Theorem 3.4. Let $\log H \geq s e^{s}$, where $s=\max \left\{e^{\kappa}+1, c_{1}+1,(m+3)^{2}+1\right\}, \kappa=[\mathbb{K}$ : $\mathbb{Q}]$. Suppose that $\lambda_{0}, \lambda_{1}, \ldots \lambda_{m} \in \mathbb{Z}_{\mathbb{K}}$ are such that at least one of them is non-zero and

$$
\prod_{v \in V_{\infty}} \max _{0 \leq i \leq m}\left\{\left\|\lambda_{i}\right\|_{v}\right\} \leq H
$$

Then there exists a prime

$$
p \in] \log \left(\frac{\log H}{\log \log H}\right), \frac{17 m \log H}{\log \log H}[
$$

and a valuation $v^{\prime} \mid p$ for which

$$
\left\|\lambda_{0}+\lambda_{1} F_{v^{\prime}}\left(\alpha_{1}\right)+\ldots+\lambda_{m} F_{v^{\prime}}\left(\alpha_{m}\right)\right\|_{v^{\prime}}>H^{-(m+1)-114 m^{2} \cdot \frac{\log \log \log H}{\log \log H}} .
$$

The idea behind the following proofs is to use Padé approximations to construct small linear forms

$$
s_{l, \mu, j}=b_{l, \mu, 0} F_{v}\left(\alpha_{j}\right)-b_{l, \mu, j}, \quad b_{l, \mu, 0}, b_{l, \mu, j} \in \mathbb{Z}_{\mathbb{K}}, \quad j=1, \ldots, m,
$$

in the numbers $F\left(\alpha_{j}\right)$. (Here $l \in \mathbb{Z}_{\geq 1}$ and $\mu \in\{0,1, \ldots, m\}$ are auxiliary parameters.) With these equations the linear form

$$
\Lambda_{v}=\lambda_{0}+\lambda_{1} F_{v}\left(\alpha_{1}\right)+\ldots+\lambda_{m} F_{v}\left(\alpha_{m}\right)
$$

under study can be written as

$$
b_{l, \mu, 0} \Lambda_{v}=W+\underset{4}{\lambda_{1} s_{l, 1}}+\ldots+\lambda_{m} s_{l, \mu, m}
$$


where $W=W(l, \mu)=\sum_{i=0}^{m} \lambda_{i} b_{l, \mu, i}$ is an integer element in $\mathbb{K}$. In case it is non-zero, the product formula implies

$$
1=\prod_{v}\|W\|_{v}
$$

In the proof of Theorem 3.1, we shall assume that $\Lambda_{v}=0$ for all $v \in V$ (whence equation (9) gives $W$ another representation as a linear combination of $s_{l, \mu, i}$ ), and then aim at a contradiction by estimating the product $\prod_{v}\|W\|_{v}$ from above. For this we need estimates for the Padé coefficients $b_{l, \mu, i}, s_{l, \mu, i}$, expressed in terms of the auxiliary parameter $l$. These are very roughly

$$
\begin{gathered}
\left\|b_{l, \mu, i}\right\|_{v} \approx(m l) !, \quad i=0,1, \ldots, m, \quad v \mid \infty, \\
\left\|s_{l, \mu, j}\right\|_{v} \approx(m l) ! l !, \quad j=1, \ldots, m, \quad v \in V_{0} .
\end{gathered}
$$

The contradiction with (10) is reached via the condition (6) when $l$ is taken to infinity.

When the target is a precise lower bound for $\left\|\Lambda_{v}\right\|_{v}$, the use of the parameter $l$ also becomes more subtle: We define the number $\ell$ so that it is the largest $l$ for which the expression

$$
N(l) \approx \log H+m l \log \log l-l \log l
$$

is still positive. Then we make the assumption that

$$
\left\|b_{\ell+1, \mu, 0} \Lambda_{v}\right\|_{v}<\left\|\lambda_{1} s_{\ell+1, \mu, 1}+\ldots+\lambda_{m} s_{\ell+1, \mu, m}\right\|_{v}
$$

for all $v \mid p, p \in[\log (\ell+1), m(\ell+2)] \cap \mathbb{P}$. This leads to the estimate

$$
0 \leq \log \left(\prod_{v}\|W(\ell+1, \mu)\|_{v}\right) \approx \log H+m(\ell+1) \log \log (\ell+1)-(\ell+1) \log (\ell+1)<0,
$$

giving the desired contradiction. It follows that there exists a prime

$$
p \in[\log (\ell+1), m(\ell+2)]
$$

and a valuation $v^{\prime} \mid p$ such that

$$
\|W(\ell+1, \mu)\|_{v^{\prime}} \leq\left\|\Lambda_{v^{\prime}}\right\|_{v^{\prime}}
$$

leading to

$$
1 \leq\left(\prod_{v \in V_{\infty}}\|W(\ell+1, \mu)\|_{v}\right)\left\|\Lambda_{v^{\prime}}\right\|_{v^{\prime}}
$$

This is the key to the lower bound for $\left\|\Lambda_{v^{\prime}}\right\|_{v^{\prime}}$, and the final step is to give an estimate for the product $\prod_{v \in V_{\infty}}\|W(\ell+1, \mu)\|_{v}$. Approximately it is

$$
\log \left(\prod_{v \in V_{\infty}}\|W(\ell+1, \mu)\|_{v}\right) \approx(m+1) \log H+m^{2} \ell \log \log \ell .
$$

The definition of $\ell$ gives a connection between $\ell$ and $H$, enabling us to write the bound (12) and the interval (111) solely in terms of $H$ :

$$
\ell \log \log \ell \approx \frac{\log \log \log H}{\log \log H} \cdot \log H .
$$

As the attentive reader may have noted, one crucial point in the proofs is the nonvanishing of the quantity $W(l, \mu)$. This is the part where the auxiliary parameter $\mu$ is needed. A non-vanishing determinant of the Padé polynomials will ensure that for each $l \in \mathbb{Z}_{\geq 1}$, there exists a $\mu \in\{0,1, \ldots, m\}$ such that $W(l, \mu) \neq 0$. 


\section{PAdÉ APproximations}

Let $m \in \mathbb{Z}_{\geq 1}, \bar{l}=\left(l_{1}, \ldots, l_{m}\right)^{T} \in \mathbb{Z}_{\geq 1}^{m}$, and $L:=\sum_{j=1}^{m} l_{j}$. For a given vector $\bar{\beta}=$ $\left(\beta_{1}, \ldots, \beta_{m}\right)^{T}$, define the numbers $\sigma_{i}=\sigma_{i}(\bar{l}, \bar{\beta})$ by the equation

$$
\prod_{j=1}^{m}\left(\beta_{j}-w\right)^{l_{j}}=\sum_{i=0}^{L} \sigma_{i} w^{i}
$$

Then, by the binomial theorem,

$$
\sigma_{i}(\bar{l}, \bar{\beta})=(-1)^{i} \sum_{i_{1}+\ldots+i_{m}=i}\left(\begin{array}{l}
l_{1} \\
i_{1}
\end{array}\right) \cdots\left(\begin{array}{l}
l_{m} \\
i_{m}
\end{array}\right) \cdot \beta_{1}^{l_{1}-i_{1}} \cdots \beta_{m}^{l_{m}-i_{m}} .
$$

Lemma 4.1. We have

$$
\sum_{i=0}^{L} \sigma_{i} i^{k} \beta_{j}^{i}=0
$$

for all $j \in\{1, \ldots, m\}, k \in\left\{0,1, \ldots, l_{j}-1\right\}$. Moreover, when $\bar{\beta}=\left(\beta_{1}, \ldots, \beta_{m}\right)^{T} \in \mathbb{K}^{m}$ and $\|\cdot\|_{v}$ is any Archimedean absolute value of the field $\mathbb{K}$, we have

$$
\sum_{i=0}^{L}\left\|\sigma_{i}\right\|_{v} t^{i} \leq \prod_{j=1}^{m}\left(\left\|\beta_{j}\right\|_{v}+t\right)^{l_{j}}
$$

for $t \geq 0$.

Proof. It is not too hard to deduce that

$$
\left(x \frac{\mathrm{d}}{\mathrm{d} x}\right)^{n} f(x)=\sum_{i=1}^{n} a_{n, i} x^{i}\left(\frac{\mathrm{d}}{\mathrm{d} x}\right)^{i} f(x),
$$

where the coefficients $a_{n, i}$ satisfy the recursions

$$
\left\{\begin{array}{l}
a_{n, 1}=1 ; \\
a_{n, i}=a_{n-1, i-1}+i a_{n-1, i}, \quad i=2, \ldots, n-1 ; \\
a_{n, n}=1
\end{array}\right.
$$

for all $n \in \mathbb{Z}_{\geq 1}$. Let now $j \in\{1, \ldots, m\}$. For $k=0$, the claim (14) follows directly from the definition (13). For $k \in\left\{1, \ldots, l_{j}-1\right\}$, we use (13) and (16):

$$
\sum_{i=0}^{L} \sigma_{i} i^{k} \beta_{j}^{i}=\left.\left(w \frac{\mathrm{d}}{\mathrm{d} w}\right)^{k} \prod_{i=1}^{m}\left(\beta_{i}-w\right)^{l_{l}}\right|_{w=\beta_{j}}=0
$$

because $\left.\left(\frac{\mathrm{d}}{\mathrm{d} w}\right)^{h} \prod_{i=1}^{m}\left(\beta_{i}-w\right)^{l_{i}}\right|_{w=\beta_{j}}=0$ for all $h \in\{1, \ldots, k\}$.

Property (15) follows simply from the expansion of $\sigma_{i}$ and the triangle inequality:

$$
\begin{aligned}
\sum_{i=0}^{L}\left\|\sigma_{i}\right\|_{v} t^{i} & \leq \sum_{i=0}^{L}\left(\sum_{i_{1}+\ldots+i_{m}=i}\left\|\left(\begin{array}{l}
l_{1} \\
i_{1}
\end{array}\right) \cdots\left(\begin{array}{c}
l_{m} \\
i_{m}
\end{array}\right)\right\|_{v} \cdot\left\|\beta_{1}\right\|_{v}^{l_{1}-i_{1}} \cdots\left\|\beta_{m}\right\|_{v}^{l_{m}-i_{m}}\right) t^{i} \\
& \leq \sum_{i=0}^{L}\left(\sum_{i_{1}+\ldots+i_{m}=i}\left(\begin{array}{c}
l_{1} \\
i_{1}
\end{array}\right) \cdots\left(\begin{array}{c}
l_{m} \\
i_{m}
\end{array}\right) \cdot\left\|\beta_{1}\right\|_{v}^{l_{1}-i_{1}} \cdots\left\|\beta_{m}\right\|_{v}^{l_{m}-i_{m}}\right) t^{i} \\
& =\prod_{j=1}^{m}\left(\left\|\beta_{j}\right\|_{v}+t\right)^{l_{j}}
\end{aligned}
$$


when $t \geq 0$.

4.1. Generalised factorial series. When $l_{1}=l_{2}=\ldots=l_{m}$, the following theorem is a particular case of Theorem 2.2 in [12]. Due to the special nature of the function (3), however, we don't need to restrict the parameters $l_{j}$.

Theorem 4.2. Let $G(t)=\sum_{n=0}^{\infty}[P]_{n} t^{n}$, where $P(x)$ is a polynomial of degree one and $[P]_{n}=\prod_{k=0}^{n-1} P(k)$. Let $\mu \in \mathbb{Z}_{\geq 0}$ and set

$$
A_{\bar{l}, \mu, 0}(t)=\sum_{i=0}^{L} \frac{\sigma_{i}(\bar{l}, \bar{\beta})}{[P]_{i+\mu}} t^{L-i}
$$

Then there exist polynomials $A_{\bar{l}, \mu, j}(t)$ and remainders $R_{\bar{l}, \mu, j}(t), j=1, \ldots, m$, such that

$$
A_{\bar{l}, \mu, 0}(t) G\left(\beta_{j} t\right)-A_{\bar{l}, \mu, j}(t)=R_{\bar{l}, \mu, j}(t),
$$

where

$$
\left\{\begin{array}{l}
\operatorname{deg} A_{\bar{l}, \mu, 0}(t)=L \\
\operatorname{deg} A_{\bar{l}, \mu, j}(t) \leq L+\mu-1, \\
\operatorname{ord} R_{\bar{l}, \mu, j}(t) \geq L+\mu+l_{j} .
\end{array}\right.
$$

Proof. Writing

$$
A_{\bar{l}, \mu, 0}(t)=\sum_{h=0}^{L} \frac{\sigma_{L-h}(\bar{l}, \bar{\beta})}{[P]_{L-h+\mu}} t^{h}
$$

we have

$$
A_{\bar{l}, \mu, 0}(t) G\left(\beta_{j} t\right)=\sum_{N=0}^{\infty} r_{N, j} t^{N}
$$

where

$$
r_{N, j}=\sum_{n+h=N} \sigma_{L-h}(\bar{l}, \bar{\beta}) \cdot \frac{[P]_{n}}{[P]_{L-h+\mu}} \cdot \beta_{j}^{n}=\sum_{h=0}^{\min \{L, N\}} \sigma_{L-h}(\bar{l}, \bar{\beta}) \cdot \frac{[P]_{N-h}}{[P]_{L-h+\mu}} \cdot \beta_{j}^{N-h} .
$$

When $N=L+\mu+a, 0 \leq a \leq l_{j}-1$, then

$$
\begin{aligned}
r_{N, j} & =\beta_{j}^{\mu+a} \sum_{h=0}^{L} \sigma_{L-h}(\bar{l}, \bar{\beta})\left(\prod_{k=1}^{a} P(L+\mu-h-1+k)\right) \beta_{j}^{L-h} \\
& =\beta_{j}^{\mu+a} \sum_{i=0}^{L} \sigma_{i}(\bar{l}, \bar{\beta})\left(\prod_{k=1}^{a} P(i+\mu-1+k)\right) \beta_{j}^{i} .
\end{aligned}
$$

(Note that the product above equals 1 when $a=0$.) Since $\operatorname{deg} P(x)=1$, we may write

$$
\prod_{k=1}^{a} P(i+\mu-1+k)=\sum_{k=0}^{a} p_{k} i^{k}
$$

where the coefficients $p_{k}$ do not depend on $i$. Hence

$$
r_{N, j}=\beta_{j}^{\mu+a} \sum_{i=0}^{L} \sigma_{i}(\bar{l}, \bar{\beta})\left(\sum_{k=0}^{a} p_{k} i^{k}\right) \beta_{j}^{i}=\beta_{j}^{\mu+a} \sum_{k=0}^{a} p_{k} \sum_{i=0}^{L} \sigma_{i}(\bar{l}, \bar{\beta}) i^{k} \beta_{j}^{i}=0
$$

due to (14). Thus we can choose

$$
A_{\bar{l}, \mu, j}(t)=\sum_{7}^{L+\mu-1} r_{N, j} t^{N}
$$


and

$$
R_{\bar{l}, \mu, j}(t)=\sum_{N=L+\mu+l_{j}}^{\infty} r_{N, j} t^{N}
$$

4.2. Euler's factorial series. To prove Theorem 3.1, we need approximations to the series $F\left(\alpha_{j} t\right)$. Thus we choose $P(x)=1+x$ and $\bar{\beta}=\bar{\alpha}=\left(\alpha_{1}, \ldots, \alpha_{m}\right)^{T}$, and set $l_{j}=l \in \mathbb{Z}_{\geq 1}$ for all $j \in\{1, \ldots, m\}$. Theorem 4.2 gives

$$
A_{\bar{l}, \mu, 0}(t)=\sum_{i=0}^{m l} \frac{\sigma_{i}}{(i+\mu) !} t^{m l-i}, \quad \sigma_{i}=\sigma_{i}(\bar{l}, \bar{\alpha}),
$$

and, directly by (20) and (19),

$$
A_{\bar{l}, \mu, j}(t)=\sum_{N=0}^{m l+\mu-1} t^{N} \sum_{h=0}^{\min \{m l, N\}} \sigma_{m l-h} \cdot \frac{(N-h) !}{(m l-h+\mu) !} \cdot \alpha_{j}^{N-h}, \quad j \in\{1, \ldots, m\} .
$$

Similarly by (21) and (19), for $N=(m+1) l+\mu+k, k \in \mathbb{N}$, we have

$$
\begin{aligned}
r_{N, j} & =\sum_{h=0}^{m l} \sigma_{m l-h} \cdot \frac{((m+1) l+\mu+k-h) !}{(m l-h+\mu) !} \cdot \alpha_{j}^{(m+1) l+\mu+k-h} \\
& =\alpha_{j}^{l+\mu+k} \sum_{i=0}^{m l} \sigma_{i} \cdot \frac{(i+\mu+l+k) !}{(i+\mu) !} \cdot \alpha_{j}^{i} \\
& =l ! k !\left(\begin{array}{c}
l+k \\
k
\end{array}\right) \alpha_{j}^{l+\mu+k} \sum_{i=0}^{m l} \sigma_{i}\left(\begin{array}{c}
i+\mu+l+k \\
i+\mu
\end{array}\right) \alpha_{j}^{i},
\end{aligned}
$$

so that

$$
R_{\bar{l}, \mu, j}(t)=l ! t^{(m+1) l+\mu} \sum_{k=0}^{\infty} t^{k} k !\left(\begin{array}{c}
l+k \\
k
\end{array}\right) \alpha_{j}^{l+\mu+k} \sum_{i=0}^{m l} \sigma_{i}\left(\begin{array}{c}
i+\mu+l+k \\
i+\mu
\end{array}\right) \alpha_{j}^{i}, \quad j=1, \ldots, m .
$$

To make the polynomials belong to $\mathbb{Z}_{\mathbb{K}}[t]$, we multiply everything by $(m l+\mu)$ ! and denote

$$
\begin{gathered}
B_{l, \mu, 0}(t):=(m l+\mu) ! A_{\bar{l}, \mu, 0}(t)=\sum_{i=0}^{m l} \sigma_{i} \cdot \frac{(m l+\mu) !}{(i+\mu) !} \cdot t^{m l-i}, \\
B_{l, \mu, j}(t):=(m l+\mu) ! A_{\bar{l}, \mu, j}(t)=(m l+\mu) ! \sum_{N=0}^{m l+\mu-1} t^{N} \sum_{h=0}^{m i n\{m l, N\}} \sigma_{m l-h} \cdot \frac{(N-h) !}{(m l-h+\mu) !} \cdot \alpha_{j}^{N-h}, \\
S_{l, \mu, j}(t):=(m l+\mu) ! R_{\bar{l}, \mu, j}(t) \\
=(m l+\mu) ! l ! t^{(m+1) l+\mu} \sum_{k=0}^{\infty} k !\left(\begin{array}{c}
l+k \\
k
\end{array}\right) \alpha_{j}^{l+k+\mu} t^{k} \sum_{i=0}^{m l} \sigma_{i}\left(\begin{array}{c}
i+\mu+l+k \\
i+\mu
\end{array}\right) \alpha_{j}^{i} .
\end{gathered}
$$

In this notation, the Padé approximation formula in (17) may be rewritten as

$$
B_{l, \mu, 0}(t) F\left(\alpha_{j} t\right)-B_{l, \mu, j}(t)=S_{l, \mu, j}(t), \quad j=1, \ldots, m .
$$




\section{LiNEAR FORM AND PRODUCT FORMULA}

Let $\lambda_{0}, \lambda_{1}, \ldots, \lambda_{m} \in \mathbb{Z}_{\mathbb{K}}$ be such that at least one of them is non-zero, and denote

$$
\Lambda_{v}:=\lambda_{0}+\lambda_{1} F_{v}\left(\alpha_{1}\right)+\ldots+\lambda_{m} F_{v}\left(\alpha_{m}\right)
$$

when $v \in V_{0}$. Equation (23) gives

$$
s_{l, \mu, i}=b_{l, \mu, 0} F_{v}\left(\alpha_{i}\right)-b_{l, \mu, i}
$$

where

$$
b_{l, \mu, i}=B_{l, \mu, i}(1), \quad i=0,1, \ldots, m ; \quad s_{l, \mu, i}=S_{l, \mu, i}(1), \quad i=1, \ldots, m .
$$

Assume that $\Lambda_{v}=0$ for all $v \in V$, where the collection $V$ satisfies condition (6). Then also

where

$$
0=b_{l, \mu, 0} \Lambda_{v}=W+\lambda_{1} s_{l, \mu, 1}+\ldots+\lambda_{m} s_{l, \mu, m}
$$

$$
W=W(l, \mu):=\lambda_{0} b_{l, \mu, 0}+\lambda_{1} b_{l, \mu, 1}+\ldots+\lambda_{m} b_{l, \mu, m} \in \mathbb{Z}_{\mathbb{K}}
$$

If $W \neq 0$, then

$$
\begin{aligned}
1 & =\prod_{v}\|W\|_{v} \leq\left(\prod_{v \in V_{\infty}}\|W\|_{v}\right) \prod_{v \in V}\|W\|_{v} \\
& \leq\left(\prod_{v \in V_{\infty}}\left\|\lambda_{0} b_{l, \mu, 0}+\lambda_{1} b_{l, \mu, 1}+\ldots+\lambda_{m} b_{l, \mu, m}\right\|_{v}\right) \prod_{v \in V}\left\|-\lambda_{1} s_{l, \mu, 1}-\ldots-\lambda_{m} s_{l, \mu, m}\right\|_{v} \\
& \leq\left(\prod_{v \in V_{\infty}}\left(\sum_{i=0}^{m}\left\|\lambda_{i}\right\|_{v}\right) \max _{0 \leq i \leq m}\left\{\left\|b_{l, \mu, i}\right\|_{v}\right\}\right) \prod_{v \in V} \max _{1 \leq i \leq m}\left\{\left\|s_{l, \mu, i}\right\|_{v}\right\} .
\end{aligned}
$$

Next we shall see that such a non-zero $W(l, \mu)$ actually exists.

\section{Determinant}

Lemma 6.1. When the numbers $\alpha_{j}, j \in\{1, \ldots, m\}$, are pairwise different and non-zero, we have

$$
\Delta(t):=\left|\begin{array}{cccc}
B_{l, 0,0}(t) & B_{l, 0,1}(t) & \cdots & B_{l, 0, m}(t) \\
B_{l, 1,0}(t) & B_{l, 1,1}(t) & \cdots & B_{l, 1, m}(t) \\
\vdots & \vdots & \ddots & \vdots \\
B_{l, m, 0}(t) & B_{l, m, 1}(t) & \cdots & B_{l, m, m}(t)
\end{array}\right| \neq 0 .
$$

Proof. By (18), the degrees of the entries are at most

$$
\left(\begin{array}{cccc}
m l & m l-1 & \cdots & m l-1 \\
m l & m l & \cdots & m l \\
\vdots & \vdots & \ddots & \vdots \\
m l & m l+m-1 & \cdots & m l+m-1
\end{array}\right)
$$

Hence

$$
\operatorname{deg} \Delta(t) \leq(m+1) m l+\frac{(m-1) m}{2} .
$$

Column operations together with (23) yield the representation

$$
\Delta(t)=\left|\begin{array}{cccc}
B_{l, 0,0}(t) & -S_{l, 0,1}(t) & \cdots & -S_{l, 0, m}(t) \\
B_{l, 1,0}(t) & -S_{l, 1,1}(t) & \cdots & -S_{l, 1, m}(t) \\
\vdots & \vdots & \ddots & \vdots \\
B_{l, m, 0}(t) & -S_{l, m, 1}(t) & \cdots & -S_{l, m, m}(t)
\end{array}\right|
$$


According to (18), the orders of the entries in (25) are at least

$$
\left(\begin{array}{cccc}
0 & (m+1) l & \cdots & (m+1) l \\
0 & (m+1) l+1 & \cdots & (m+1) l+1 \\
\vdots & \vdots & \ddots & \vdots \\
0 & (m+1) l+m & \cdots & (m+1) l+m
\end{array}\right)
$$

By expanding (25) by the first column we see that

$$
\operatorname{ord} \Delta(t) \geq \sum_{i=0}^{m-1}((m+1) l+i)=m(m+1) l+\frac{(m-1) m}{2} .
$$

Thus

$$
\Delta(t)=b t^{m(m+1) l+\frac{(m-1) m}{2}},
$$

where the coefficient $b$ is an $m \times m$ determinant formed from the lowest term coefficients of the remainders $-S_{l, \mu, j}, \mu=0,1, \ldots, m-1, j=1, \ldots, m$ (corresponding to $k=0$ in (22) $)$, multiplied by the lowest term coefficient of the polynomial $B_{l, m, 0}(t)$ which is $\sigma_{m l}=(-1)^{m l}$ :

$$
\begin{gathered}
b=(-1)^{m l} \cdot(-1)^{m}(l !)^{m}\left(\prod_{\mu=0}^{m-1}(m l+\mu) !\right)\left(\prod_{j=1}^{m} \alpha_{j}^{l}\right) \\
\left|\begin{array}{cccc}
\sum_{i=0}^{m l} \sigma_{i}\left(\begin{array}{c}
i+l \\
i
\end{array}\right) \alpha_{1}^{i} & \sum_{i=0}^{m l} \sigma_{i}\left(\begin{array}{c}
i+l \\
i
\end{array}\right) \alpha_{2}^{i} & \cdots & \sum_{i=0}^{m l} \sigma_{i}\left(\begin{array}{c}
i+l \\
i
\end{array}\right) \alpha_{m}^{i} \\
\alpha_{1} \sum_{i=0}^{m l} \sigma_{i}\left(\begin{array}{c}
i+1+l \\
i+1
\end{array}\right) \alpha_{1}^{i} & \alpha_{2} \sum_{i=0}^{m l} \sigma_{i}\left(\begin{array}{c}
i+1+l \\
i+1
\end{array}\right) \alpha_{2}^{i} & \cdots & \alpha_{m} \sum_{i=0}^{m l} \sigma_{i}\left(\begin{array}{c}
i+1+l \\
i+1
\end{array}\right) \alpha_{m}^{i} \\
\vdots & \vdots & \ddots & \vdots \\
\alpha_{1}^{m-1} \sum_{i=0}^{m l} \sigma_{i}\left(\begin{array}{c}
i+m-1+l \\
i+m-1
\end{array}\right) \alpha_{1}^{i} & \alpha_{2}^{m-1} \sum_{i=0}^{m l} \sigma_{i}\left(\begin{array}{c}
i+m-1+l \\
i+m-1
\end{array}\right) \alpha_{2}^{i} & \cdots & \alpha_{m}^{m-1} \sum_{i=0}^{m l} \sigma_{i}\left(\begin{array}{c}
i+m-1+l \\
i
\end{array}\right) \alpha_{m}^{i}
\end{array}\right| .
\end{gathered}
$$

It remains to show that $b \neq 0$.

Since

$$
\left(\begin{array}{c}
i+\mu+l \\
i+\mu
\end{array}\right)=\frac{(i+\mu+l) !}{(i+\mu) ! l !}=\frac{1}{l !}(i+\mu+1) \cdots(i+\mu+l)=\frac{1}{l !}\left(i^{l}+\sum_{k=0}^{l-1} p_{k} i^{k}\right)
$$

for any $\mu \in\{0,1, \ldots, m-1\}$, where the coefficients $p_{k}$ do not depend on $i$, we get

$$
\sum_{i=0}^{m l} \sigma_{i}\left(\begin{array}{c}
i+\mu+l \\
i+\mu
\end{array}\right) \alpha_{j}^{i}=\frac{1}{l !}\left(\sum_{i=0}^{m l} \sigma_{i} i^{l} \alpha_{j}^{i}+\sum_{k=0}^{l-1} p_{k} \sum_{i=0}^{m l} \sigma_{i} i^{k} \alpha_{j}^{i}\right)=\frac{1}{l !} \sum_{i=0}^{m l} \sigma_{i} i^{l} \alpha_{j}^{i}
$$

for all $j=1, \ldots, m, \mu=0,1, \ldots, m-1$ by the property (14). Hence

$$
\begin{aligned}
& b=(-1)^{m(l+1)}\left(\prod_{\mu=0}^{m-1}(m l+\mu) !\right)\left(\prod_{j=1}^{m} \alpha_{j}^{l}\right) . \\
& \left|\begin{array}{cccc}
\sum_{i=0}^{m l} \sigma_{i} i^{l} \alpha_{1}^{i} & \sum_{i=0}^{m l} \sigma_{i} i^{l} \alpha_{2}^{i} & \cdots & \sum_{i=0}^{m l} \sigma_{i} i^{l} \alpha_{m}^{i} \\
\alpha_{1} \sum_{i=0}^{m l} \sigma_{i} l^{l} \alpha_{1}^{i} & \alpha_{2} \sum_{i=0}^{m l} \sigma_{i} i^{l} \alpha_{2}^{i} & \cdots & \alpha_{m} \sum_{i=0}^{m l} \sigma_{i} i^{l} \alpha_{m}^{i} \\
\vdots & \vdots & \ddots & \vdots \\
\alpha_{1}^{m-1} \sum_{i=0}^{m l} \sigma_{i} i^{l} \alpha_{1}^{i} & \alpha_{2}^{m-1} \sum_{i=0}^{m l} \sigma_{i} i^{l} \alpha_{2}^{i} & \cdots & \alpha_{m}^{m-1} \sum_{i=0}^{m l} \sigma_{i} i^{l} \alpha_{m}^{i}
\end{array}\right| \\
& =(-1)^{m(l+1)}\left(\prod_{\mu=0}^{m-1}(m l+\mu) !\right)\left(\prod_{j=1}^{m} \alpha_{j}^{l}\right)\left(\prod_{10}^{m}\left(\sum_{j=1}^{m l} \sigma_{i} i^{l} \alpha_{j}^{i}\right)\right) \prod_{1 \leq i<j \leq m}\left(\alpha_{j}-\alpha_{i}\right)
\end{aligned}
$$


by the Vandermonde determinant formula. Here, using (13) and (16),

$$
\sum_{i=0}^{m l} \sigma_{i} i^{l} \alpha_{j}^{i}=\left.\left(w \frac{\mathrm{d}}{\mathrm{d} w}\right)^{l} \prod_{i=1}^{m}\left(\alpha_{i}-w\right)^{l}\right|_{w=\alpha_{j}}=(-1)^{l} l ! \alpha_{j}^{l} \prod_{\substack{i=1 \\ i \neq j}}^{m}\left(\alpha_{i}-\alpha_{j}\right)^{l} \neq 0
$$

for all $j=1, \ldots, m$.

Lemma 6.2. For any given $l \in \mathbb{Z}_{\geq 1}$ there exists a $\mu \in\{0,1, \ldots, m\}$ such that $W(l, \mu) \neq$ 0 .

Proof. From Lemma 6.1 it follows in particular that

$$
\left|\begin{array}{cccc}
b_{l, 0,0} & b_{l, 0,1} & \cdots & b_{l, 0, m} \\
b_{l, 1,0} & b_{l, 1,1} & \cdots & b_{l, 1, m} \\
\vdots & \vdots & \ddots & \vdots \\
b_{l, m, 0} & b_{l, m, 1} & \cdots & b_{l, m, m}
\end{array}\right|=\Delta(1) \neq 0
$$

We assumed that $\left(\lambda_{0}, \lambda_{1}, \ldots, \lambda_{m}\right)^{T} \neq \overline{0}$, so by linear algebra it follows that the quantity $W(l, \mu)=\lambda_{0} b_{l, \mu, 0}+\lambda_{1} b_{l, \mu, 1}+\ldots+\lambda_{m} b_{l, \mu, m}$ must be non-zero for some $\mu \in\{0,1, \ldots, m\}$.

7. Estimates For the POlynomials AND REMAinders AND PROOF OF THEOREM 3.1

As the last step in proving Theorem 3.1, we give upper bounds for the Padé polynomials and remainders. Now, using the triangle inequality and property (15) with $v \mid \infty$,

$$
\begin{aligned}
& \left\|b_{l, \mu, 0}\right\|_{v}=\left\|B_{l, \mu, 0}(1)\right\|_{v}=\left\|\sum_{i=0}^{m l} \sigma_{i} \frac{(m l+\mu) !}{(i+\mu) !}\right\|_{v} \\
& \leq\left\|(m l) !\left(\begin{array}{c}
m l+\mu \\
\mu
\end{array}\right)\right\|_{v} \sum_{i=0}^{m l}\left\|\sigma_{i}\right\|_{v} \leq\left\|(m l) !\left(\begin{array}{c}
m l+\mu \\
\mu
\end{array}\right)\right\|_{v} \prod_{j=1}^{m}\left(\left\|\alpha_{j}\right\|_{v}+1\right)^{l}
\end{aligned}
$$


and

$$
\begin{aligned}
\left\|b_{l, \mu, j}\right\|_{v} & =\left\|B_{l, \mu, j}(1)\right\|_{v} \\
& =\left\|(m l+\mu) ! \sum_{N=0}^{m l+\mu-1} \sum_{h=0}^{\min \{m l, N\}} \frac{(N-h) !}{(m l-h+\mu) !} \sigma_{m l-h} \alpha_{j}^{N-h}\right\|_{v} \\
\leq & \|(m l+\mu) !\|_{v} \sum_{N=0}^{m l+\mu-1} \sum_{h=0}^{\min \{m l, N\}}\left\|\frac{(N-h) !}{(m l-h+\mu) !}\right\|_{v}\left\|\sigma_{m l-h}\right\|_{v}\left\|\alpha_{j}\right\|_{v}^{N-h} \\
\leq & \|(m l+\mu) !\|_{v} \sum_{N=0}^{m l+\mu-1} \sum_{h=0}^{\min \{m l, N\}}\left\|\sigma_{m l-h}\right\|_{v}\left(\max \left\{1,\left\|\alpha_{j}\right\|_{v}\right\}\right)^{N-h} \\
\leq & \|(m l+\mu) !\|_{v} \sum_{N=0}^{m l+\mu-1} \sum_{h=0}^{\min \{m l, N\}}\left\|\sigma_{m l-h}\right\|_{v}\left(\max \left\{1,\left\|\alpha_{j}\right\|_{v}\right\}\right)^{m l+m-1-h} \\
\leq & \|(m l+\mu) !\|_{v}\left(\max \left\{1,\left\|\alpha_{j}\right\|_{v}\right\}\right)^{m-1}(m l+m) \cdot \\
& \quad \sum_{h=0} \sum_{i n}\left\{\sigma_{m l, N} \|_{v}\left(\max \left\{1,\left\|\alpha_{j}\right\|_{v}\right\}\right)^{m l-h}\right. \\
\leq & \|(m l+\mu) !\|_{v}\left(\max \left\{1,\left\|\alpha_{j}\right\|_{v}\right\}\right)^{m l}(m l+m) \prod_{i=1}^{m}\left(\left\|\alpha_{i}\right\|_{v}+\max \left\{1,\left\|\alpha_{j}\right\|_{v}\right\}\right)^{l}
\end{aligned}
$$

for all $j=1, \ldots, m, \mu=0,1, \ldots, m$.

We still need non-Archimedean estimates for the remainders, so let now $v \in V_{0}$. Then

$$
\begin{aligned}
\left\|s_{l, \mu, j}\right\|_{v} & =\left\|S_{l, \mu, j}(1)\right\|_{v} \\
& =\left\|(m l+\mu) ! l ! \sum_{k=0}^{\infty} k !\left(\begin{array}{c}
l+k \\
k
\end{array}\right) \alpha_{j}^{l+k+\mu} \sum_{i=0}^{m l} \sigma_{i}\left(\begin{array}{c}
i+\mu+l+k \\
i+\mu
\end{array}\right) \alpha_{j}^{i}\right\|_{v} \\
& \leq\|(m l+\mu) ! l !\|_{v}\left\|\alpha_{j}\right\|_{v}^{l} .
\end{aligned}
$$

for all $j=1, \ldots, m, \mu=0,1, \ldots, m$.

So, recalling property (5) of our normalised valuations, the expression in (24) becomes

$$
\begin{aligned}
& \left(\prod_{v \in V_{\infty}}\left(\sum_{i=0}^{m}\left\|\lambda_{i}\right\|_{v}\right) \max _{0 \leq i \leq m}\left\{\left\|b_{l, \mu, i}\right\|_{v}\right\}\right) \prod_{v \in V} \max _{1 \leq i \leq m}\left\{\left\|s_{l, \mu, i}\right\|_{v}\right\} \\
\leq & \left(\prod_{v \in V_{\infty}}\left(\sum_{i=0}^{m}\left\|\lambda_{i}\right\|_{v}\right)(m l+m)\|(m l+m) !\|_{v}\left(\max _{1 \leq j \leq m}\left\{1,\left\|\alpha_{j}\right\|_{v}\right\}\right)^{m l} \cdot\right. \\
& \left.\prod_{i=1}^{m}\left(\left\|\alpha_{i}\right\|_{v}+\max _{1 \leq j \leq m}\left\{1,\left\|\alpha_{j}\right\|_{v}\right\}\right)^{l}\right) \cdot \prod_{v \in V}\|(m l) ! l !\|_{v}\left(\max _{1 \leq j \leq m}\left\{\left\|\alpha_{j}\right\|_{v}\right\}\right)^{l} \\
\leq & \left(\prod_{v \in V_{\infty}}\left(\sum_{i=0}^{m}\left\|\lambda_{i}\right\|_{v}\right)\right) c_{2}^{l}(m l+m)^{\kappa}(m l+m) ! \cdot \prod_{v \in V}\|(m l) ! l !\|_{v},
\end{aligned}
$$


where

$$
\begin{aligned}
c_{2}= & \left(\prod_{v \in V_{\infty}}\left(\left(\max _{1 \leq j \leq m}\left\{1,\left\|\alpha_{j}\right\|_{v}\right\}\right)^{m} \prod_{i=1}^{m}\left(\left\|\alpha_{i}\right\|_{v}+\max _{1 \leq j \leq m}\left\{1,\left\|\alpha_{j}\right\|_{v}\right\}\right)\right)\right) . \\
& \prod_{v \in V} \max _{1 \leq j \leq m}\left\{\left\|\alpha_{j}\right\|_{v}\right\} .
\end{aligned}
$$

Proof of Theorem 3.1. In Section [6 we saw that for every $l \in \mathbb{Z}_{>1}$, there exists a $\mu \in$ $\{0,1, \ldots, m\}$ such that $W=W(l, \mu) \neq 0$. Hence the estimate in (24) holds for infinitely many $W(l, \mu)$, so that our assumption $\Lambda_{v}=0$ for all $v \in V$ and estimates (24) and (26) lead to

$$
1 \leq\left(\prod_{v \in V_{\infty}}\left(\sum_{i=0}^{m}\left\|\lambda_{i}\right\|_{v}\right)\right) c_{2}^{l}(m l+m)^{\kappa}(m l+m) ! \prod_{v \in V}\|(m l) ! l !\|_{v}
$$

which holds for infinitely many $l$. This is a contradiction with condition (6), and thus there must exist a valuation $v^{\prime} \in V$ such that $\Lambda_{v^{\prime}} \neq 0$.

\section{LOWER BOUND: PROOF OF THEOREM 3.4}

8.1. Product formula again. The fundamental product formula (44) is the starting point for the proof of our second theorem as well. We repeat Section 5 with a slightly more refined assumption. First we need some notation though.

Let $m \in \mathbb{Z}_{\geq 1}$ and $\log H \geq s e^{s}$, where

$$
\begin{gathered}
s=\max \left\{e^{\kappa}+1, c_{1}+1,(m+3)^{2}+1\right\} \\
\kappa=[\mathbb{K}: \mathbb{Q}], \\
c_{1}=\prod_{v \in V_{\infty}}\left(\left(\max _{1 \leq j \leq m}\left\{1,\left\|\alpha_{j}\right\|_{v}\right\}\right)^{m} \prod_{i=1}^{m}\left(\left\|\alpha_{i}\right\|_{v}+\max _{1 \leq j \leq m}\left\{1,\left\|\alpha_{j}\right\|_{v}\right\}\right)\right) .
\end{gathered}
$$

Suppose that $\lambda_{0}, \lambda_{1}, \ldots, \lambda_{m} \in \mathbb{Z}_{\mathbb{K}}$ are such that at least one of them is non-zero and

$$
\prod_{v \in V_{\infty}} \max _{0 \leq i \leq m}\left\{\left\|\lambda_{i}\right\|_{v}\right\} \leq H
$$

Define

$$
\begin{aligned}
N(l):= & \log H+\left(2(m+1)+\frac{2 m}{l}+\frac{\log c_{1}}{\log \log l}+\frac{1}{\log \log l}+\frac{\left(\kappa-\frac{1}{2}\right) \log l}{l \log \log l}\right. \\
& \left.+\frac{\kappa \log m}{l \log \log l}+\frac{\kappa \log (m+1)}{l \log \log l}+\frac{\kappa}{l^{2} \log \log l}\right) l \log \log l-l \log l
\end{aligned}
$$

and let

$$
\ell:=\max \left\{l \in \mathbb{Z}_{\geq 2} \mid N(l) \geq 0\right\}
$$

Denote, as before,

$$
\Lambda_{v}=\lambda_{0}+\lambda_{1} F_{v}\left(\alpha_{1}\right)+\ldots+\lambda_{m} F_{v}\left(\alpha_{m}\right) .
$$

We saw in Section 5 that

$$
b_{l, \mu, 0} \Lambda_{v}=W+\lambda_{1} s_{l, \mu, 1}+\ldots+\lambda_{m} s_{l, \mu, m}
$$

where

$$
W=W(l, \mu)=\lambda_{0} b_{l, \mu, 0}+\lambda_{1} b_{l, \mu, 1}+\ldots+\lambda_{m} b_{l, \mu, m} \in \mathbb{Z}_{\mathbb{K}}
$$


By Lemma 6.2 we know that $W(\ell+1, \mu) \neq 0$ for some $\mu \in\{0,1, \ldots, m\}$. Assume that

$$
\left\|b_{\ell+1, \mu, 0} \Lambda_{v}\right\|_{v}<\left\|\lambda_{1} s_{\ell+1, \mu, 1}+\ldots+\lambda_{m} s_{\ell+1, \mu, m}\right\|_{v}
$$

for all $v \mid p, p \in[\log (\ell+1), m(\ell+2)] \cap \mathbb{P}$. (The intersection certainly is non-empty due to Bertrand's postulate. As for the choice of this interval, see Remark 8.5.) Then

$$
\begin{aligned}
\|W(\ell+1, \mu)\|_{v} & =\left\|b_{\ell+1, \mu, 0} \Lambda_{v}-\left(\lambda_{1} s_{\ell+1, \mu, 1}+\ldots+\lambda_{m} s_{\ell+1, \mu, m}\right)\right\|_{v} \\
& =\left\|\lambda_{1} s_{\ell+1, \mu, 1}+\ldots+\lambda_{m} s_{\ell+1, \mu, m}\right\|_{v}
\end{aligned}
$$

for all $v \mid p, p \in[\log (\ell+1), m(\ell+2)] \cap \mathbb{P}$. Hence, using the estimates made in Section 7 together with property (5),

$$
\begin{aligned}
& 1=\prod_{v}\|W(\ell+1, \mu)\|_{v} \\
& \leq\left(\prod_{v \in V_{\infty}}\|W\|_{v}\right)_{p \in[\log (\ell+1), m(\ell+2)]} \prod_{v \mid p}\|W\|_{v} \\
& =\left(\prod_{v \in V_{\infty}}\left\|\sum_{i=0}^{m} \lambda_{i} b_{\ell+1, \mu, i}\right\|_{v}\right) \prod_{p \in[\log (\ell+1), m(\ell+2)]} \prod_{v \mid p}\left\|\lambda_{1} s_{\ell+1, \mu, 1}+\ldots+\lambda_{m} s_{\ell+1, \mu, m}\right\|_{v} \\
& \leq\left(\prod_{v \in V_{\infty}}(m+1) \max _{0 \leq i \leq m}\left\{\left\|\lambda_{i}\right\|_{v}\right\} \max _{0 \leq i \leq m}\left\{\left\|b_{\ell+1, \mu, i}\right\|_{v}\right\}\right) \text {. } \\
& \prod_{p \in[\log (\ell+1), m(\ell+2)]} \prod_{v \mid p} \max _{1 \leq i \leq m}\left\{\left\|s_{\ell+1, \mu, i}\right\|_{v}\right\} \\
& \leq(m+1)^{\kappa} H\left(\prod _ { v \in V _ { \infty } } \left((m(\ell+1)+m)\|(m(\ell+1)+\mu) !\|_{v} .\right.\right. \\
& \left.\left.\left(\max _{1 \leq j \leq m}\left\{1,\left\|\alpha_{j}\right\|_{v}\right\}\right)^{m(\ell+1)} \prod_{i=1}^{m}\left(\left\|\alpha_{i}\right\|_{v}+\max _{1 \leq j \leq m}\left\{1,\left\|\alpha_{j}\right\|_{v}\right\}\right)^{\ell+1}\right)\right) . \\
& \prod_{p \in[\log (\ell+1), m(\ell+2)]} \prod_{v \mid p}\|(m(\ell+1)+\mu) !(\ell+1) !\|_{v} \\
& \leq(m+1)^{\kappa}(m(\ell+1)+m)^{\kappa} H c_{1}^{\ell+1}(m(\ell+1)+\mu) ! . \\
& \prod_{p \in[\log (\ell+1), m(\ell+2)]}|(m(\ell+1)+\mu) !(\ell+1) !|_{p} \\
& =\frac{(m+1)^{\kappa}(m(\ell+2))^{\kappa} H c_{1}^{\ell+1} \prod_{p \in[\log (\ell+1), m(\ell+2)]}|(\ell+1) !|_{p}}{\prod_{p<\log (\ell+1)}|(m(\ell+1)+\mu) !|_{p}}=: \Omega,
\end{aligned}
$$

where we utilised the fact that $\# V_{\infty} \leq \kappa=[\mathbb{K}: \mathbb{Q}]$. The last equality is due to the product formula and the fact $m(\ell+1)+\mu \leq m(\ell+2)$. 
8.2. Deriving contradiction. We are working to establish a contradiction with (30), so let us study the expression $\log \Omega$ more closely. First of all, we have

$$
\begin{aligned}
\Omega & =\frac{(m+1)^{\kappa}(m(\ell+2))^{\kappa} H c_{1}^{\ell+1} \prod_{p \in[\log (\ell+1), m(\ell+2)]}|(\ell+1) !|_{p}}{\prod_{p<\log (\ell+1)}|(m(\ell+1)+\mu) !|_{p}} \\
& =\frac{(m+1)^{\kappa}(m(\ell+2))^{\kappa} H c_{1}^{\ell+1}}{(\ell+1) ! \prod_{p<\log (\ell+1)}|(m(\ell+1)+\mu) !(\ell+1) !|_{p}} \\
& \leq \frac{(m+1)^{\kappa}(m(\ell+2))^{\kappa} H c_{1}^{\ell+1} \prod_{p<\log (\ell+1)} p^{\frac{m(\ell+1)+\mu+(\ell+1)}{p-1}}}{(\ell+1) !}
\end{aligned}
$$

because of the product formula and property (7). Recall also the Stirling formula

$$
\log n !=\left(n+\frac{1}{2}\right) \log n-n+\log \sqrt{2 \pi}+\frac{\theta(n)}{12}, \quad 0<\theta(n)<1 .
$$

With these equations and estimate $\mu \leq m$ we get

$$
\begin{aligned}
& \log \Omega \leq \log \left(\frac{(m+1)^{\kappa}(m(\ell+2))^{\kappa} H c_{1}^{\ell+1} \prod_{p<\log (\ell+1)} p^{\frac{m(\ell+1)+\mu+(\ell+1)}{p-1}}}{(\ell+1) !}\right) \\
& \leq \kappa \log (m+1)+\kappa \log (m(\ell+2))+\log H+(\ell+1) \log c_{1}+ \\
& \sum_{p<\log (\ell+1)} \log p^{\frac{m(\ell+2)+(\ell+1)}{p-1}}-\left((\ell+1)+\frac{1}{2}\right) \log (\ell+1)+(\ell+1) \\
& \leq \kappa \log (m+1)+\kappa \log m+\kappa \log (\ell+2)+\log H+(\ell+1) \log c_{1} \\
& +(m(\ell+2)+(\ell+1)) \sum_{p<\log (\ell+1)} \frac{\log p}{p-1}-(\ell+1) \log (\ell+1) \\
& -\frac{1}{2} \log (\ell+1)+(\ell+1) \\
& \leq \log H+\kappa \log m+\kappa \log (m+1)+\frac{\kappa}{\ell+1}+\left(\kappa-\frac{1}{2}\right) \log (\ell+1) \\
& +\left(\log c_{1}+\left(m+1+\frac{m}{\ell+1}\right) \sum_{p<\log (\ell+1)} \frac{\log p}{p-1}+1\right)(\ell+1) \\
& -(\ell+1) \log (\ell+1) \text {, }
\end{aligned}
$$

where

$$
\log (\ell+2)<\log (\ell+1)+\frac{1}{\ell+1}
$$

by the mean value theorem.

To be able to continue, we need to know how the sum

$$
\sum_{p<x} \frac{\log p}{p-1}
$$

behaves. Help is found from [13] (see the corollary of Theorem 6): 
Lemma 8.1. [13]

$$
\sum_{p \leq x} \frac{\log p}{p}<\log x, \quad x>1 .
$$

Since $p-1 \geq \frac{p}{2}$ for all primes $p$, it follows that

$$
\sum_{p<x} \frac{\log p}{p-1} \leq 2 \sum_{p<x} \frac{\log p}{p}<2 \log x .
$$

Combining estimates (30), (311), and (32), we have

$$
\begin{aligned}
0 \leq & \log \Omega \\
\leq & \log H+\kappa \log m+\kappa \log (m+1)+\frac{\kappa}{\ell+1}+\left(\kappa-\frac{1}{2}\right) \log (\ell+1) \\
& +\left(\log c_{1}+\left(m+1+\frac{m}{\ell+1}\right) \sum_{p<\log (\ell+1)} \frac{\log p}{p-1}+1\right)(\ell+1)-(\ell+1) \log (\ell+1) \\
< & \log H+\kappa \log m+\kappa \log (m+1)+\frac{\kappa}{\ell+1}+\left(\kappa-\frac{1}{2}\right) \log (\ell+1) \\
& +\left(\log c_{1}+2\left(m+1+\frac{m}{\ell+1}\right) \log \log (\ell+1)+1\right)(\ell+1)-(\ell+1) \log (\ell+1) \\
< & \log H+\left(2(m+1)+\frac{2 m}{\ell+1}+\frac{\log c_{1}}{\log \log (\ell+1)}+\frac{1}{\log \log (\ell+1)}\right. \\
& +\frac{\left(\kappa-\frac{1}{2}\right) \log (\ell+1)}{(\ell+1) \log \log (\ell+1)}+\frac{\kappa \log m}{(\ell+1) \log \log (\ell+1)}+\frac{\kappa \log (m+1)}{(\ell+1) \log \log (\ell+1)} \\
& \left.+\frac{\kappa}{(\ell+1)^{2} \log \log (\ell+1)}\right)(\ell+1) \log \log (\ell+1)-(\ell+1) \log (\ell+1) \\
= & N(\ell+1)<0,
\end{aligned}
$$

a contradiction with (29). Thus there must exist a prime

$$
p \in[\log (\ell+1), m(\ell+2)]
$$

and a valuation $v^{\prime} \mid p$ such that $\left\|b_{\ell+1, \mu, 0} \Lambda_{v^{\prime}}\right\|_{v^{\prime}} \geq\left\|\lambda_{1} s_{\ell+1, \mu, 1}+\ldots+\lambda_{m} s_{\ell+1, \mu, m}\right\|_{v^{\prime}}$. Then, for this valuation $v^{\prime}$,

$$
\|W\|_{v^{\prime}}=\left\|b_{\ell+1, \mu, 0} \Lambda_{v^{\prime}}-\left(\lambda_{1} s_{\ell+1, \mu, 1}+\ldots+\lambda_{m} s_{\ell+1, \mu, m}\right)\right\|_{v^{\prime}} \leq\left\|b_{\ell+1, \mu, 0} \Lambda_{v^{\prime}}\right\|_{v^{\prime}} \leq\left\|\Lambda_{v^{\prime}}\right\|_{v^{\prime}}
$$

and

$$
1=\prod_{v}\|W\|_{v} \leq\left(\prod_{v \in V_{\infty}}\|W\|_{v}\right)\|W\|_{v^{\prime}} \leq\left(\prod_{v \in V_{\infty}}\|W\|_{v}\right)\left\|\Lambda_{v^{\prime}}\right\|_{v^{\prime}} .
$$

8.3. Bounds for $\ell$. For the final stages of the proof, we need to express the number $\ell$ in terms of the height $H$. In order to do this, we introduce the inverse function of the function $y(z)=z \log z, z \geq 1 / e$, considered in [9].

Lemma 8.2. 9] The inverse function $z(y)$ of the function $y(z)=z \log z, z \geq 1 / e$, is strictly increasing. Define $z_{0}(y)=y$ and $z_{n}(y)=\frac{y}{\log z_{n-1}(y)}$ for $n \in \mathbb{Z}_{\geq 1}$. Suppose $y>e$, 
then $z_{1}<z_{3}<\cdots<z<\cdots<z_{2}<z_{0}$. Thus the inverse function may be given by the infinite nested logarithm fraction

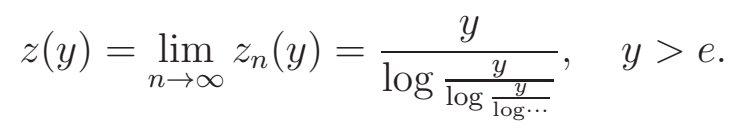

Another little lemma from [7] gives a useful upper estimate:

Lemma 8.3. [7] If $y \geq r e^{r}$, where $r \geq e$, then

$$
z(y) \leq\left(1+\frac{\log r}{r}\right) \frac{y}{\log y} .
$$

Proof. Denote $z:=z(y)$ with $y \geq r e^{r}$. Then

$$
z=\frac{y}{\log z}=\frac{y}{\log y} \frac{\log y}{\log z}=\frac{y}{\log y}\left(1+\frac{\log \log z}{\log z}\right) \leq \frac{y}{\log y}\left(1+\frac{\log r}{r}\right),
$$

because $\log z \geq r \geq e$.

Now, $N(\ell+1)<0$ implies

$$
(\ell+1) \log (\ell+1)>\log H \geq s e^{s},
$$

so that (applying the $z$-function) $\ell+1>e^{s}$. According to (27), we have

$$
\ell>e^{s}-1 \geq \max \left\{e^{e^{\kappa}}, e^{c_{1}}, e^{(m+3)^{2}}\right\}
$$

Hence, using the lower bound (36) and the fact that $m \geq 1$, we may estimate from the definition of $N(l)$ in (28):

$$
\begin{aligned}
0 \leq & N(\ell) \\
< & \log H+\left(2(m+1)+\frac{2 m}{e^{(m+3)^{2}}}+1+\frac{1}{2 \log (m+3)}+\frac{\left(\kappa-\frac{1}{2}\right)(m+3)^{2}}{e^{(m+3)^{2}} \cdot \kappa}\right. \\
& \left.+\frac{\kappa \log m}{e^{(m+3)^{2}} \cdot \kappa}+\frac{\kappa \log (m+1)}{e^{(m+3)^{2}} \cdot \kappa}+\frac{\kappa}{e^{2(m+3)^{2}} \cdot \kappa}\right) \ell \log \log \ell-\ell \log \ell \\
\leq & \log H+\left(2(m+1)+1+0.360674+3 \cdot 10^{-6}\right) \ell \log \log \ell-\ell \log \ell \\
< & \log H+(2 m+3.361) \ell \log \log \ell-\ell \log \ell .
\end{aligned}
$$

Thus

$$
\ell \log \ell\left(1-\frac{(2 m+3.361) \log \log \ell}{\log \ell}\right) \leq \log H
$$

where

$$
\frac{\log \log \ell}{\log \ell}<\frac{2 \log (m+3)}{(m+3)^{2}}
$$

by (36), and so

$$
1-\frac{(2 m+3.361) \log \log \ell}{\log \ell}>1-\frac{(2 m+3.361) \cdot 2 \log (m+3)}{(m+3)^{2}}>0
$$

for all $m \geq 1$.

By inequalities (38) and (39) and the lower bound in (36), we have

$$
\frac{(m+3)^{2}}{(m+3)^{2}-(2 m+3.361) \cdot 2 \log (m+3)} \cdot \log H>\ell \log \ell>(m+3)^{2} e^{(m+3)^{2}},
$$


so we may apply Lemma 8.3 with $r=(m+3)^{2}$ :

$$
\begin{aligned}
\ell & <z\left(\frac{(m+3)^{2}}{(m+3)^{2}-(2 m+3.361) \cdot 2 \log (m+3)} \cdot \log H\right) \\
& \leq\left(1+\frac{2 \log (m+3)}{(m+3)^{2}}\right) \frac{\frac{(m+3)^{2}}{(m+3)^{2}-(2 m+3.361) \cdot 2 \log (m+3)} \cdot \log H}{\log \log H} .
\end{aligned}
$$

8.4. Measure. To get the measure from (34), we need an upper estimate for the product $\prod_{v \in V_{\infty}}\|W\|_{v}$. Back in (30) we estimated that

$$
\prod_{v \in V_{\infty}}\|W\|_{v} \leq(m+1)^{\kappa}(m(\ell+2))^{\kappa} H c_{1}^{\ell+1}(m(\ell+2)) !
$$

(taking into account that $\mu \leq m$ ). From (37) it follows that

$$
\ell \log \ell<(2 m+3.361) \ell \log \log \ell+\log H
$$

and by the mean value theorem we have

$$
\log (\ell+2)<\frac{2}{\ell}+\log \ell
$$

With these estimates we get

$$
\begin{aligned}
& \log \left(\prod_{v \in V_{\infty}}\|W\|_{v}\right) \\
& \leq \log \left((m+1)^{\kappa}(m(\ell+2))^{\kappa} H c_{1}^{\ell+1}(m(\ell+2)) !\right) \\
& \leq \kappa \log (m+1)+\kappa \log m+\kappa \log (\ell+2)+\log H+(\ell+1) \log c_{1} \\
& +(m(\ell+2)) \log (m(\ell+2)) \\
& =\kappa \log (m+1)+\kappa \log m+\kappa \log (\ell+2)+\log H+\ell \log c_{1}+\log c_{1} \\
& +(m \log m) \ell+m \ell \log (\ell+2)+2 m \log m+2 m \log (\ell+2) \\
& \leq \kappa \log (m+1)+\kappa \log m+\frac{2 \kappa}{\ell}+\kappa \log \ell+\log H+\ell \log c_{1}+\log c_{1}+(m \log m) \ell \\
& +m \ell \log \ell+2 m+2 m \log m+2 m \log \ell+\frac{4 m}{\ell} \\
& <\kappa \log (m+1)+\kappa \log m+\frac{2 \kappa}{\ell}+\kappa \log \ell+\log H+\ell \log c_{1}+\log c_{1}+(m \log m) \ell \\
& +m((2 m+3.361) \ell \log \log \ell+\log H)+2 m+2 m \log m+2 m \log \ell+\frac{4 m}{\ell} \\
& =(m+1) \log H+\left(\frac{\kappa \log (m+1)}{\ell \log \log \ell}+\frac{\kappa \log m}{\ell \log \log \ell}+\frac{2 \kappa}{\ell^{2} \log \log \ell}+\frac{\kappa \log \ell}{\ell \log \log \ell}\right. \\
& +\frac{\log c_{1}}{\log \log \ell}+\frac{\log c_{1}}{\ell \log \log \ell}+\frac{m \log m}{\log \log \ell}+2 m^{2}+3.361 m+\frac{2 m}{\ell \log \log \ell} \\
& \left.+\frac{2 m \log m}{\ell \log \log \ell}+\frac{2 m \log \ell}{\ell \log \log \ell}+\frac{4 m}{\ell^{2} \log \log \ell}\right) \ell \log \log \ell
\end{aligned}
$$

In the coefficient of $\ell \log \log \ell$, we have (using the bound (36) and the fact that $m \geq 1$ )

$$
\frac{m \log m}{\log \log \ell}<\frac{m \log m}{2 \log (m+3)}<\frac{m}{2}, \quad \frac{\log c_{1}}{\log \log \ell}<1,
$$


and the rest of the fractions together are less than 0.0000034 . Hence

$$
\log \left(\prod_{v \in V_{\infty}}\|W\|_{v}\right) \leq(m+1) \log H+\left(2 m^{2}+3.861 m+1.0000034\right) \ell \log \log \ell
$$

By (40) and the assumption $\log H \geq s e^{s}>(m+3)^{2} e^{(m+3)^{2}}$, we have

$$
\begin{aligned}
\ell & <\frac{\left(1+\frac{2 \log (m+3)}{(m+3)^{2}}\right)(m+3)^{2}}{(m+3)^{2}-(2 m+3.361) \cdot 2 \log (m+3)} \cdot \frac{\log H}{2 \log (m+3)+(m+3)^{2}} \\
& =\frac{1}{(m+3)^{2}-(2 m+3.361) \cdot 2 \log (m+3)} \cdot \log H<\log H .
\end{aligned}
$$

Thus

$$
\log \log \ell<\log \log \log H
$$

Let us next estimate $\left(2 m^{2}+3.861 m+1.0000034\right) \ell$, again using (40):

$$
\begin{aligned}
& \left(2 m^{2}+3.861 m+1.0000034\right) \ell \\
\leq & \frac{\left(2 m^{2}+3.861 m+1.0000034\right)\left(1+\frac{2 \log (m+3)}{(m+3)^{2}}\right)(m+3)^{2}}{(m+3)^{2}-(2 m+3.361) \cdot 2 \log (m+3)} \cdot \frac{\log H}{\log \log H} \\
= & \frac{m^{2}\left(2+\frac{3.861}{m}+\frac{1.0000034}{m^{2}}\right)\left(1+\frac{2 \log (m+3)}{(m+3)^{2}}\right)}{1-\frac{2 \cdot 2 m \log (m+3)}{(m+3)^{2}}-\frac{2 \cdot 3.361 \log (m+3)}{(m+3)^{2}}} \cdot \frac{\log H}{\log \log H} \\
< & 114 m^{2} \cdot \frac{\log H}{\log \log H}
\end{aligned}
$$

since $m \geq 1$.

Combining estimates (41), (42), and (43), yields

$$
\log \left(\prod_{v \in V_{\infty}}\|W\|_{v}\right)<\left((m+1)+114 m^{2} \cdot \frac{\log \log \log H}{\log \log H}\right) \log H,
$$

so that inequality (34) implies

$$
\left\|\Lambda_{v^{\prime}}\right\|_{v^{\prime}} \geq \frac{1}{\prod_{v \in V_{\infty}}\|W\|_{v}}>H^{-(m+1)-114 m^{2} \cdot \frac{\log \log \log H}{\log \log H}}
$$

8.5. Infinitely many intervals. We still need an upper estimate for $m(\ell+1)$ in terms of the height $H$ in order to write the interval (33) with respect to $H$. Once more we use 
(40) and the assumption $\log H \geq s e^{s}>(m+3)^{2} e^{(m+3)^{2}}$ :

$$
\begin{aligned}
& m(\ell+2) \\
\leq & m \cdot \frac{\left(1+\frac{2 \log (m+3)}{(m+3)^{2}}\right)(m+3)^{2}}{(m+3)^{2}-(2 m+3.361) \cdot 2 \log (m+3)} \cdot \frac{\log H}{\log \log H}+2 m \\
= & m\left(\frac{1+\frac{2 \log (m+3)}{(m+3)^{2}}}{1-\frac{2 \cdot 2 m \log (m+3)}{(m+3)^{2}}-\frac{2 \cdot 3.361 \log (m+3)}{(m+3)^{2}}}+\frac{2 \log \log H}{\log H}\right) \cdot \frac{\log H}{\log \log H} \\
\leq & m\left(\frac{1+\frac{2 \log (m+3)}{(m+3)^{2}}}{1-\frac{2 \cdot 2 m \log (m+3)}{(m+3)^{2}}-\frac{2 \cdot 3.361 \log (m+3)}{(m+3)^{2}}}+\frac{4 \log (m+3)+2(m+3)^{2}}{(m+3)^{2} e^{(m+3)^{2}}}\right) \cdot \frac{\log H}{\log \log H} \\
< & 17 m \cdot \frac{\log H}{\log \log H}
\end{aligned}
$$

since $m \geq 1$.

By (35) and Lemma 8.3 we have

$$
\log (\ell+1)>\log (z(\log H))>\log \left(z_{1}(\log H)\right)=\log \left(\frac{\log H}{\log \log H}\right) .
$$

Combining this with (44) above leads to

$$
[\log (\ell+1), m(\ell+2)] \subseteq] \log \left(\frac{\log H}{\log \log H}\right), \frac{17 m \log H}{\log \log H}[=: I(m, H) .
$$

Letting $H$ have values in a very rapidly increasing sequence, something like $H_{i+1}=e^{e^{H_{i}}}$, the intervals $I\left(m, H_{i}\right)$ will be distinct.

This ends the proof of Theorem 3.4 .

Remark 8.4. The constants 114 and 17 can be improved by adjusting the lower bound of $\log H$, i.e. the choice of $s$ in (27). For instance, taking $(m+3)^{3}$ instead of $(m+3)^{2}$ will reduce them considerably.

Remark 8.5. There is a connection between the width of the interval $I(m, H)$ and the error term in the lower bound (8). Our choice of $\log (\ell+1)$ in the interval (33) results in the term $\log \log \log H$ in (8) (see (42)), improving the corresponding lower bound of Bertrand et. al. in 2 for this function. This is done at a cost, though, since our interval $I(m, H)$ is wider than theirs. Had we chosen $e^{\sqrt{\log (\ell+1)}}$ instead of $\log (\ell+1)$, we would have ended up with $\sqrt{\log \log H}$ instead of $\log \log \log H$. Then the dependence on $H$ in the error term of (8) would have been $\frac{1}{\sqrt{\log \log H}}$, just as it is in [2], and the interval $I(m, H)$ would have had $\exp \left(\sqrt{\log \left(\frac{\log H}{\log \log H}\right)}\right)$ as its lower bound, very much like in [2] and [14].

The best lower bound (in terms of $H$ ) would have been achieved by considering an interval of the form $[2, m l]$ with no dependence on $l$ in the lower bound, because the empty sum $\sum_{p<2} \frac{\log p}{p-1}$ would not then cause an extra term in our estimates. The disappearing of $\log \log l$ from the estimates would mean that we would have $\frac{1}{\log \log H}$ instead of $\frac{\log \log \log H}{\log \log H}$ in the error term. This is in line with the exponential function (see [7]). However, this result won't give us infinitely many distinct primes when $H$ grows, like Theorem 3.4 does. 


\section{Corollaries And examples}

9.1. The field of rationals. When $\mathbb{K}=\mathbb{Q}$, Theorem 3.1 reduces to:

Corollary 9.1. Let $m \in \mathbb{Z}_{\geq 1}$ and $\lambda_{0}, \lambda_{1}, \ldots, \lambda_{m} \in \mathbb{Z}$ where $\lambda_{j} \neq 0$ for at least one $j$. Choose $m$ pairwise distinct, non-zero integers $\alpha_{j} \in \mathbb{Z} \backslash\{0\}, j=1, \ldots, m$. Suppose $P$ is a subset of the prime numbers such that

$$
\limsup _{l \rightarrow \infty} c_{2}^{l}(m l+m)(m l+m) ! \prod_{p \in P}|(m l) ! l !|_{p}=0,
$$

where

$$
c_{2}=\left(\max _{1 \leq j \leq m}\left\{1,\left|\alpha_{j}\right|\right\}\right)^{m}\left(\prod_{i=1}^{m}\left(\left|\alpha_{i}\right|+\max _{1 \leq j \leq m}\left\{1,\left|\alpha_{j}\right|\right\}\right)\right) \prod_{p \in P} \max _{1 \leq j \leq m}\left\{\left|\alpha_{j}\right|_{p}\right\} .
$$

Then there exists a prime $p^{\prime} \in P$ for which

$$
\lambda_{0}+\lambda_{1} F_{p^{\prime}}\left(\alpha_{1}\right)+\ldots+\lambda_{m} F_{p^{\prime}}\left(\alpha_{m}\right) \neq 0 .
$$

Example 9.2. For instance, take $\alpha_{1}=1$ and $\alpha_{2}=-1$. Then, if $P \subseteq \mathbb{P}$ is such that

$$
\limsup _{l \rightarrow \infty} 4^{l}(2 l+2)(2 l+2) ! \prod_{p \in P}|(2 l) ! l !|_{p}=0,
$$

there exists a prime $p^{\prime} \in P$ for which

$$
\lambda_{0}+\lambda_{1} F_{p^{\prime}}(1)+\lambda_{2} F_{p^{\prime}}(-1) \neq 0 .
$$

In particular, taking $\lambda_{0}=2 a \in \mathbb{Z}$ and $\lambda_{1}=\lambda_{2}=-b \in \mathbb{Z}$, it follows that there exists a prime $p \in P$ such that

$$
a-b \sum_{n=0}^{\infty}(2 n) ! \neq 0
$$

i.e. $\sum_{n=0}^{\infty}(2 n) ! \neq \frac{a}{b}$ for some $p^{\prime} \in P$.

9.2. Linear recurrences. A sequence $\left(x_{n}\right)_{n=0}^{\infty}$ satisfies a $k$ th order homogeneous linear recurrence with constant coefficients, if, for all $n \in \mathbb{Z}_{\geq k}$,

$$
x_{n}=c_{1} x_{n-1}+c_{2} x_{n-2}+\ldots+c_{k} x_{n-k}
$$

for some $c_{1}, \ldots, c_{k} \in \mathbb{C}$ with $c_{k} \neq 0$. If the characteristic polynomial $x^{k}-c_{1} x^{k-1}-\ldots-c_{k} \in$ $\mathbb{C}[x]$ of this recurrence has $k$ distinct zeros $\alpha_{1}, \ldots, \alpha_{k} \in \mathbb{C}$, then the solution $\left(x_{n}\right)_{n=0}^{\infty}$ is given by the linear combination

$$
x_{n}=a_{1} \alpha_{1}^{n}+\ldots+a_{k} \alpha_{k}^{n}, \quad n \in \mathbb{Z}_{\geq 0},
$$

where the coefficients $a_{1}, \ldots, a_{k} \in \mathbb{C}$ are determined by given initial conditions. (More about recurrences in [6].)

Suppose now that $c_{1}, \ldots, c_{k} \in \mathbb{Z}$. Then the roots $\alpha_{1}, \ldots, \alpha_{k}$ lie in a number field $\mathbb{K}$ of degree at most $k$, and so do the coefficients $a_{1}, \ldots, a_{k}$. Furthermore, if $\alpha_{1}, \ldots, \alpha_{k} \in \mathbb{Z}_{\mathbb{K}}$, then $F\left(\alpha_{i}\right), i=1, \ldots, k$, converges for any non-Archimedean valuation $v$ of $\mathbb{K}$, and we have

$$
\sum_{i=1}^{k} a_{i} F_{v}\left(\alpha_{i}\right)=\sum_{i=1}^{k} a_{i} \sum_{n=0}^{\infty} n ! \alpha_{i}^{n}=\sum_{n=0}^{\infty} n ! \sum_{i=1}^{k} a_{i} \alpha_{i}^{n}=\sum_{n=0}^{\infty} n ! x_{n} .
$$


Multiplying both sides by $d:=\operatorname{lcm}_{1 \leq i \leq k}\left\{\operatorname{den} a_{i}\right\}$ 2we get a linear form with coefficients $b_{i}:=d a_{i} \in \mathbb{Z}_{\mathbb{K}}:$

$$
\sum_{i=1}^{k} b_{i} F_{v}\left(\alpha_{i}\right)=d \sum_{n=0}^{\infty} n ! x_{n}
$$

If at least one of the coefficients $a_{i}$ is non-zero, it follows from Theorem 3.1 that for any $a, b \in \mathbb{Z}_{\mathbb{K}}$ there exists a non-Archimedean valuation $v^{\prime}$ of $\mathbb{K}$ such that

$$
\sum_{n=0}^{\infty} n ! x_{n} \neq \frac{a}{b}
$$

Example 9.3 (The Fibonacci numbers). The Fibonacci numbers are given by the sequence

$$
f_{n}=\frac{1}{\sqrt{5}}\left(\alpha^{n}-\beta^{n}\right), \quad \alpha=\frac{1+\sqrt{5}}{2}, \quad \beta=\frac{1-\sqrt{5}}{2}, \quad n \in \mathbb{Z}_{\geq 0} .
$$

Let us work in $\mathbb{Q}(\sqrt{5})$ and study the series $\sum_{n=0}^{\infty} n ! f_{n}$. The minimal polynomial of $\alpha$ and $\beta$ is $x^{2}-x-1$, so $\alpha$ and $\beta$ are algebraic integers and thus $\|\alpha\|_{v},\|\beta\|_{v} \leq 1$ for any non-Archimedean valuation $v$ of the field $\mathbb{Q}(\sqrt{5})$. Actually, as $\alpha \beta=-1$, we get $\|\alpha\|_{v}=\|\beta\|_{v}=1$ for all $v \in V_{0}$. Hence both series $\frac{1}{\sqrt{5}} \sum_{n=0}^{\infty} n ! \alpha^{n}$ and $-\frac{1}{\sqrt{5}} \sum_{n=0}^{\infty} n ! \beta^{n}$ converge $v$-adically and their sum is

$$
\frac{1}{\sqrt{5}}\left(F_{v}(\alpha)-F_{v}(\beta)\right)=\frac{1}{\sqrt{5}}\left(\sum_{n=0}^{\infty} n ! \alpha^{n}-\sum_{n=0}^{\infty} n ! \beta^{n}\right)=\frac{1}{\sqrt{5}} \sum_{n=0}^{\infty} n !\left(\alpha^{n}-\beta^{n}\right)=\sum_{n=0}^{\infty} n ! f_{n} .
$$

Because $x^{2}-5=(x-\sqrt{5})(x+\sqrt{5})$ in $\mathbb{R}[x]$, the Archimedean absolute value of $\mathbb{Q}$ has two extensions to $\mathbb{Q}(\sqrt{5})$. These are given by

$$
|a+b \sqrt{5}|_{1}=|a+b \sqrt{5}|, \quad|a+b \sqrt{5}|_{2}=|a-b \sqrt{5}|,
$$

where now $|\cdot|$ is the unique Archimedean extension of the Archimedean absolute value of $\mathbb{Q}$ to $\mathbb{C}$, the algebraic closure of the Archimedean completion of $\mathbb{Q}$. Further,

$$
\begin{aligned}
& \|a+b \sqrt{5}\|_{1}=|a+b \sqrt{5}|_{1}^{\frac{1}{2}}=\sqrt{|a+b \sqrt{5}|}, \\
& \|a+b \sqrt{5}\|_{2}=|a+b \sqrt{5}|_{2}^{\frac{1}{2}}=\sqrt{|a-b \sqrt{5}|} .
\end{aligned}
$$

Let $a, b \in \mathbb{Z}, b \neq 0$ and choose $\alpha_{1}=\alpha, \alpha_{2}=\beta$. Then

$$
\begin{aligned}
c_{2}((\alpha, \beta), V)= & \left(\max \left\{1,\|\alpha\|_{1},\|\beta\|_{1}\right\}\right)^{2}\left(\max \left\{1,\|\alpha\|_{2},\|\beta\|_{2}\right\}\right)^{2} . \\
& \left(\|\alpha\|_{1}+\max \left\{1,\|\alpha\|_{1},\|\beta\|_{1}\right\}\right)\left(\|\beta\|_{1}+\max \left\{1,\|\alpha\|_{1},\|\beta\|_{1}\right\}\right) . \\
& \left(\|\alpha\|_{2}+\max \left\{1,\|\alpha\|_{2},\|\beta\|_{2}\right\}\right)\left(\|\beta\|_{2}+\max \left\{1,\|\alpha\|_{2},\|\beta\|_{2}\right\}\right) . \\
& \prod_{v \in V} \max \left\{\|\alpha\|_{v},\|\beta\|_{v}\right\} \\
= & 4\left(\frac{1+\sqrt{5}}{2}\right)^{3}\left(\sqrt{\frac{-1+\sqrt{5}}{2}}+\sqrt{\frac{1+\sqrt{5}}{2}}\right)^{2} \approx 72 .
\end{aligned}
$$

By taking $\lambda_{0}=5 a \in \mathbb{Z}, \lambda_{1}=\lambda_{2}=-b \sqrt{5} \in \mathbb{Z}_{\mathbb{K}} \backslash\{0\}$, Theorem 3.1 gives:

\footnotetext{
${ }^{2}$ The denominator den $\alpha$ of an algebraic number $\alpha \in \mathbb{K}$ is the smallest positive rational integer $n$ such that $n \alpha$ is an algebraic integer.
} 
Corollary 9.4. If $V$ is any collection of non-Archimedean valuations of $\mathbb{Q}(\sqrt{5})$ such that

$$
\limsup _{l \rightarrow \infty} c_{2}^{l}(2 l+2)(2 l+2) ! \prod_{v \in V}\|(2 l) ! l !\|_{v}=0
$$

then there exists a valuation $v^{\prime} \in V$ for which

$$
a-b \sum_{n=0}^{\infty} n ! f_{n} \neq 0
$$

9.3. Arithmetic progressions. In [8] the authors prove:

Proposition 9.5. [8, Theorem 3] Let $a \in \mathbb{Z}, b, \xi \in \mathbb{Z} \backslash\{0\}$, and $n \in \mathbb{Z}_{\geq 3}$ be given. Assume that $R$ is any union of the primes in $r$ residue classes in the reduced residue system modulo $n$, where $r>\frac{\varphi(n)}{2}$. Then there are infinitely many primes $p \in R$ such that $a-b F_{p}(\xi) \neq 0$.

Because each non-Archimedean valuation of the number field $\mathbb{K}$ is attached to the prime it extends, the division of primes into $\varphi(n)$ residue classes induces a division of the non-Archimedean valuations into $\varphi(n)$ classes. How many of these classes are needed to fulfil condition (6) ?

Theorem 9.6. Let $m \in \mathbb{Z}_{\geq 1}$ and $\lambda_{0}, \lambda_{1}, \ldots, \lambda_{m} \in \mathbb{Z}_{\mathbb{K}}$ where $\lambda_{j} \neq 0$ for at least one $j$. Choose $m$ pairwise distinct, non-zero algebraic integers $\alpha_{1}, \ldots, \alpha_{m} \in \mathbb{Z}_{\mathbb{K}}$. Let $n \in \mathbb{Z}_{\geq 3}$ be given. Assume that $R$ is a union of the primes in $r$ residue classes in the reduced residue system modulo $n$, where $r>\frac{m \varphi(n)}{m+1}$, and let $V=\left\{v \in V_{0}|v| p\right.$ for some $\left.p \in R\right\}$. Then there exists a valuation $v^{\prime} \in V$ such that

$$
\lambda_{0}+\lambda_{1} F_{v^{\prime}}\left(\alpha_{1}\right)+\ldots+\lambda_{m} F_{v^{\prime}}\left(\alpha_{m}\right) \neq 0 .
$$

Proof. Let us show that the collection $V$ satisfies condition (66). We shall follow the method in [8]. By [8, Lemma 1] we have

$$
\log \left(\prod_{p \equiv a}|l !|_{p}\right)=-\frac{l \log l}{\varphi(n)}+O(l \log \log l)
$$

when $n \in \mathbb{Z}_{\geq 3}$ and $\operatorname{gcd}(a, n)=1$. Using this, the fact

$$
\log ((m l+m) !)=m l \log l+O(l),
$$


and property (5), we get

$$
\begin{aligned}
& \log \left(c_{2}^{l}(m l+m)^{\kappa}(m l+m) ! \prod_{v \in V}\|(m l) ! l !\|_{v}\right) \\
= & l \log c_{2}+\kappa \log (m(l+1))+\log ((m l+m) !)+\sum_{v \in V} \log \|(m l) ! l !\|_{v} \\
= & m l \log l+O(l)+\sum_{p \in R} \sum_{v \mid p} \log \|(m l) ! l !\|_{v} \\
= & m l \log l+O(l)+\sum_{p \in R} \log |(m l) ! l !|_{p} \\
= & m l \log l+O(l)-\frac{r m l \log l}{\varphi(n)}-\frac{r l \log l}{\varphi(n)}+O(l \log \log l) \\
= & \left(m-\frac{r(m+1)}{\varphi(n)}\right) l \log l+O(l \log \log l) \\
l \rightarrow \infty & \rightarrow-\infty,
\end{aligned}
$$

because the coefficient $\left(m-\frac{r(m+1)}{\varphi(n)}\right)$ is negative. The result follows from Theorem 3.1 ,

\section{ACKNOWLEDGEMENTS}

The work of the author was supported by the University of Oulu Scholarship Foundation and the Vilho, Yrjö and Kalle Väisälä Foundation.

\section{REFERENCES}

[1] G. Bachman: Introduction to $p$-adic numbers and valuation theory, Academic Press, New York, 1964.

[2] D. Bertrand, V. G. Chirskiǔ, J. Yebbou: Effective estimates for global relations on Euler-type series, Ann. Fac. Sci. Toulouse Math. (6) 13 (2004), no. 2, 241-260.

[3] V. G. Chirskiǔ: Non-trivial global relations, Vestn. Mosk. Univ. Ser. I Mat. Mekh. 44 (1989), no. 5, 33-36; English translation in Moscow Univ. Math. Bull. 44 (1989), no. 5, 41-44.

[4] V. G. Chirskiı̌: Global relations, Mat. Zametki 48, No. 2, (1990), 123-127; English translation in Math. Notes 48 (1990), no. 1-2, 795-798.

[5] V. G. Chirskiŭ: On Algebraic Relations in Non-Archimedean Fields, Funct. Anal. Appl. 26 (1992), no. 2, 108-115.

[6] P. Cull, M. Flahive, R. Robson: Difference Equations, Springer, New York, 2005.

[7] A-M. Ernvall-Hytönen, T. Matala-aho, L. Seppälä: On Mahler's Transcendence Measure for $e$, Constr. Approx. (2018). https://doi.org/10.1007/s00365-018-9429-3.

[8] A-M. Ernvall-Hytönen, T. Matala-aho, L. Seppälä: Euler's divergent series in arithmetic progressions, 2018. Submitted; preprint available as arXiv:1809.03859 [math.NT].

[9] J. Hančl, M. Leinonen, K. Leppälä, T. Matala-aho: Explicit irrationality measures for continued fractions, J. Number Theory 132 (2012), 1758-1769.

[10] S. Lang: Algebraic Number Theory, Springer-Verlag, New York, 1986.

[11] T. Matala-aho, W. Zudilin: Euler's factorial series and global relations, J. Number Theory 186 (2018), 202-210.

[12] T. Matala-aho: Type II Hermite-Padé approximations of generalized hypergeometric series, Constr. Approx. 33 (2011), 289-312.

[13] J. B. Rosser, L. Schoenfeld: Approximate formulas for some functions of prime numbers, Illinois J. Math. 6 (1962), 6-94.

[14] K. Väänänen: On Padé approximations and global relations of some Euler-type series, Int. J. Number Theory 14, no. 8, (2018), 2303-2315. 
Louna Seppälä, Matematilkka, PL 8000, 90014 Oulun Yliopisto, Finland E-mail address: louna.seppala@oulu.fi 